J. Austral. Math. Soc. (Series A) 42 (1987), 84-91

\title{
SEMIDIRECT PRODUCT GROUPS WITH ABELIAN AUTOMORPHISM GROUPS
}

\author{
M. J. CURRAN \\ (Received 16 December 1983; revised 23 September 1985) \\ Communicated by D. E. Taylor
}

\begin{abstract}
Miller's group of order 64 is a smallest example of a nonabelian group with an abelian automorphism group, and is the first in an infinite family of such groups formed by taking the semidirect product of a cyclic group of order $2^{m}(m \geqslant 3)$ with a dihedral group of order 8 . This paper gives a method for constructing further examples of non abelian 2-groups which! have abelian automorphism groups. Such a 2-group is the semidirect product of a cyclic group and a special 2-group (satisfying certain conditions). The automorphism group of this semidirect product is shown to be isomorphic to the central automorphism group of the corresponding direct product. The conditions satisfied by the special 2-group are determined by establishing when this direct product has an abelian central automorphism group.
\end{abstract}

1980 Mathematics subject classification (Amer. Math. Soc.): 20 F 28.

\section{Introduction}

Any group $G$ mentioned in this paper is finite. An automorphism $\phi$ of $G$ is central if $\phi$ commutes with every inner automorphism of $G$, or equivalently if $g^{-1} g^{\phi}$ lies in the centre $Z(G)$ of $G$ for all $g \in G$. The central automorphisms form a normal subgroup, denoted $\mathrm{Aut}_{c} G$, of the full automorphism group Aut $G$. If $H$ is a subgroup of $G$, we let $\operatorname{Aut}(G: H)=\left\{\phi \in \operatorname{Aut} G \mid H^{\phi}=H\right\}$.

Struik [8] showed that Miller's group [6] of order 64 constitutes the first in an infinite family $M=\left\langle a, b, c \mid a^{2^{m}}=b^{4}=c^{2}=1, a^{b}=a^{1+2^{m-1}}, b^{c}=b^{-1}, a^{c}=a\right\rangle$, $m \geqslant 3$, of nonabelian 2-groups having abelian automorphism groups. $M$ is a

(C) 1987 Australian Mathematical Society 0263-6115/87 \$A2.00+0.00 
semidirect product of a cyclic group $A=\langle a\rangle$ of order $2^{m}$ by $N=\langle b, c\rangle \approx D_{4}$, the dihedral group of order 8 . This paper provides a method of constructing $M$ and similar semidirect product groups with abelian automorphism groups. In Section 4 we prove:

THEOREM. Let $N$ be a nonabelian special 2-group with a subgroup $J$ of index 2 such that (i) $Z(J)=Z(N)$; (ii) $\operatorname{Aut}(N: J)=$ Aut $_{c} N$. If $G=A N$ is the semidirect product of a cyclic group $A$ of order $2^{m}$ and a group $N$ such that $a^{n}=a$ if $n \in J$ and $a^{n}=a^{1+2^{m-1}}$ if $n \in N \backslash J$, then Aut $G$ is abelian.

Section 2 gives preliminary results and Section 3 gives a criterion for Aut ${ }_{c} G$ to be abelian when $G$ is a $p$-group with an abelian direct factor.

\section{Automorphisms of semidirect products}

Let $G=A N$ be a semidirect product of a group $A$ by a group $N$, with multiplication given by $(a, n)\left(a_{1}, n_{1}\right)=\left(a a_{1}^{n^{-1}}, n n_{1}\right)$, where $a, a_{1} \in A$ and $n, n_{1}$ $\in N$. We denote by $\iota_{A}$ and $\iota_{N}$ the inclusion maps from $A$ and $N$, respectively, into $G$, and by $\pi_{A}$ and $\pi_{N}$ the projection maps from $G$ to $A$ and $N$, respectively. For $\phi \in \operatorname{End} G=\operatorname{Hom}(G, G)$, we put $\alpha=\iota_{A} \phi \pi_{A}, \beta=\iota_{A} \phi \pi_{N}, \gamma=\iota_{N} \phi \pi_{A}$ and $\delta=\iota_{N} \phi \pi_{N}$. Then $\beta \in \operatorname{Hom}(A, N)$ and $\delta \in \operatorname{End} N$.

If $\phi$ also satisfies (a) $\left[A^{\beta}, A\right]=1=\left[N^{\gamma}, N\right]$, then $\alpha \in \operatorname{End} A, \gamma \in \operatorname{Hom}(N, A)$ and $(a, n)^{\phi}=\left(a^{\alpha} n^{\gamma}, a^{\beta} n^{\delta}\right)$, where $a \in A, n \in N$. If every $\phi \in$ End $G$ satisfies (a) and (b) $\left[A^{\alpha}, N^{\gamma}\right]=1=\left[A^{\beta}, N^{\delta}\right]$, and if $\mathscr{R}$ denotes the matrix semigroup

$$
\mathscr{R}=\left\{\left(\begin{array}{cc}
\alpha & \beta \\
\gamma & \delta
\end{array}\right) \mid \begin{array}{l}
\alpha \in \operatorname{End} A, \beta \in \operatorname{Hom}(A, N),\left[A^{\alpha}, N^{\gamma}\right]=1 \\
\gamma \in \operatorname{Hom}(N, A), \delta \in \operatorname{End} N,\left[A^{\beta}, N^{\delta}\right]=1
\end{array}\right\},
$$

then $f$ : End $G \rightarrow \mathscr{R}$ defined by $f(\phi)=\left(\begin{array}{cc}\alpha & \beta \\ \gamma & \delta\end{array}\right)$ is a monomorphism.

In particular, if $G=A \times N$, then every $\phi \in$ End $G$ satisfies (a) and (b) above. Conversely, if $\left(\begin{array}{cc}\alpha & \beta \\ \gamma & \beta\end{array}\right) \in \mathscr{R}$ and $\phi: G \rightarrow G$ is defined by $(a, n)^{\phi}=\left(a^{\alpha} n^{\gamma}, n^{\beta} n^{\delta}\right)$, where $a \in A, n \in N$, then $\phi \in$ End $G$. We thus have

Lemma 2.1. If $G=A \times N$, then End $G \approx \mathscr{R}$.

For convenience let

$$
\begin{aligned}
& \mathscr{L}=\left\{\left(\begin{array}{ll}
\alpha & 0 \\
\gamma & \delta
\end{array}\right) \mid \alpha \in \text { Aut } A, \gamma \in \operatorname{Hom}(N, Z(A)), \delta \in \text { Aut } N\right\}, \\
& \mathscr{H}=\left\{\left(\begin{array}{ll}
\alpha & \beta \\
\gamma & \delta
\end{array}\right) \mid \begin{array}{l}
\alpha \in \operatorname{Aut} A, \beta \in \operatorname{Hom}(A, Z(N)) \\
\gamma \in \operatorname{Hom}(N, A), \delta \in \operatorname{Aut} N
\end{array}\right\},
\end{aligned}
$$


and let $\mathscr{L}_{c}$ and $\mathscr{H}_{c}$ be the corresponding sets of matrices, where $\alpha \in$ Aut $_{c} A$ and $\delta \in$ Aut $_{c} N$.

The following two lemmas, whose proofs are straightforward, are used in Section 3.

LEMMA 2.2. Let $G=A \times N$. Then

(i) $\operatorname{Aut}(G: A) \approx \mathscr{L}$;

(ii) Aut ${ }_{c}(G: A)=\left\{\phi \in\right.$ Aut $\left._{c} G \mid A^{\phi}=A\right\} \approx \mathscr{L}_{c}$;

(iii) $\operatorname{Aut}(G: N)$ and $\operatorname{Aut}_{c}(G: N)$ are isomorphic to analogous groups of upper triangular matrices.

Lemma 2.3. Let $G=A \times N$, where $A$ is abelian. If there is a characteristic subgroup $C$ of $G$ such that $N \geqslant C \geqslant Z(N)$, then $f$ : Aut $G \rightarrow \mathscr{H}$ is a monomorphism.

The next two results are used in Section 4.

THEOREM 2.4. Let $G=A N$ be a semidirect product, where

(i) $A$ is abelian, $N^{\prime} \geqslant Z(N)$ and $[Z(N), A]=1$;

(ii) for all $\phi \in$ Aut $G, A^{\beta} \leqslant Z(N)$ and $\left[N^{\gamma}, N\right]=1$.

Then $f$ : Aut $G \rightarrow \mathscr{H}$ is a monomorphism.

Proof. From (i) and (ii), every $\phi \in$ Aut $G$ satisfies (a) and (b) above. Further, if $(a, 1)^{\phi}=(1, n)$, where $a \in A, n \in N$, then $a^{\beta}=n \in Z(N) \leqslant N^{\prime}$. But $N^{\prime}$ is characteristic in $G$, since $\left[N^{\gamma}, N\right]=1$. Thus $(1, n)^{\phi^{-1}}=(a, 1)$ implies that $a=n$ $=1$, so $\alpha \in$ Aut $A$ and $\delta \in$ Aut $N$.

THEOREM 2.5. Let $G=A \times N$, where $A$ is abelian and $N^{\prime} \geqslant Z(N)$. Then

(i) Aut $G \approx \mathscr{H}$, and

(ii) Aut $_{c} G \approx \mathscr{H}_{c}$.

Proof. (i) The conditions of Theorem 2.4 hold, so $\left.f\right|_{\text {Aut } G}$ : Aut $G \rightarrow \mathscr{H}$ is a

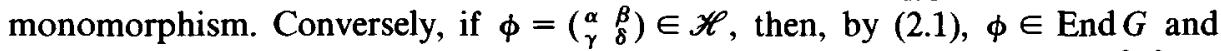
$f(\phi)=\phi$. Further, if $(a, n)^{\phi}=(1,1)$, where $a \in A, n \in N$, then $\left(a^{\alpha} n^{\gamma}, a^{\beta} n^{\delta}\right)=$ $(1,1)$. Thus $\left(a^{-1}\right)^{\beta \delta^{-1}} \in Z(N) \leqslant N^{\prime} \leqslant \operatorname{ker} \gamma$, so that $n^{\gamma}=1$. Hence $a=n=1$, and $\phi \in$ Aut $G$. Thus $f$ is an isomorphism.

(ii) $\phi \in$ Aut $_{c} G \Leftrightarrow(a, n)^{-1}(a, n)^{\phi} \in A \times Z(N) \Leftrightarrow n^{-1} n^{\delta} \in Z(N) \Leftrightarrow \delta \in$ Aut $_{c} N$. 
COROllary 2.6. If $G=A N$ satisfies the hypotheses of Theorem 2.4, then Aut $G$ is isomorphic to a subgroup of $\operatorname{Aut}(A \times N)$.

\section{Central automorphisms of groups with abelian direct factors}

The following theorem is a modification of a result of Earnley (Theorem 2.3 in [3]), but the proof is given for convenience.

ThEOREM 3.1. Let $G=A \times N$ be a p-group, where $1 \neq A$ is abelian, and where $1 \neq N$ is purely nonabelian (i.e. $N$ has no non-trivial abelian direct factor). Then Aut ${ }_{c} G$ is abelian if and only if $A$ and $N$ satisfy

(i) $A$ is cyclic of order $2^{m}, m \geqslant 2$;

(ii) $N$ is a 2-group with Aut ${ }_{c} N$ abelian;

(iii) $\Phi(N)=N^{\prime} \geqslant Z(N)$, and $Z(N)$ has exponent 2 .

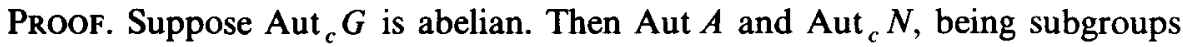
of Aut ${ }_{c} G$, are abelian. In particular, $A$ is cyclic. (See 3.12 in [2].) If $A=\langle a\rangle$, define $\tau \in$ Aut $A$ by $a^{\tau}=a^{-1}$. Thus, by Lemma 2.2, if $y \in Y=\operatorname{Hom}(N, A)$, and if $x \in X=\operatorname{Hom}(A, Z(N))$, then $\left(\begin{array}{ll}\tau & 0 \\ y & 1\end{array}\right)$ and $\left(\begin{array}{ll}1 & x \\ 0 & 1\end{array}\right)$ are in Aut ${ }_{c} G$ and so commute. Hence $x y=0=y x$ and $\tau x=x$. Now $X \neq 0$ since $A$ and $Z(N)$ are non-trivial $p$-groups. Thus $|A| \neq 2$, for otherwise there is an epimorphism $y \in Y$, and the condition $y x=0$ implies that $x=0$ for all $x \in X$. Further, $\tau x=x$ implies that $A^{x}$ has exponent dividing 2, so either $p=2$, or, again, $x=0$ for all $x \in X$.

Therefore $|A|=2^{m}, m \geqslant 2$, and $Z(N)$ has exponent 2. Hence $Z(N) \leqslant \Phi(N)$, for otherwise there exists $n_{1} \in Z(N) \backslash \Phi(N)$ of order 2 such that $\left\{n_{1}, \ldots, n_{t}\right\}$, $t \geqslant 2$, is a minimal generating set for $N$. But then $N=\left\langle n_{1}\right\rangle \times\left\langle n_{2}, \ldots, n_{t}\right\rangle$, a contradiction.

Similarly, the commutativity of $\left(\begin{array}{ll}1 & 0 \\ y & 1\end{array}\right)$ and $\left(\begin{array}{ll}\tau & x \\ 0 & 1\end{array}\right)$ gives $y \tau=y$, and hence $N^{y}$ has exponent dividing 2 . Since $A$ has exponent greater than $2, N / N^{\prime}$ has exponent 2. Thus $\Phi(N)=N^{\prime}$, and $A$ and $N$ satisfy the given conditions.

Conversely, suppose that $A$ and $N$ satisfy (i), (ii) and (iii). Then, by Theorem 2.5, Aut ${ }_{c} G \approx \mathscr{H}_{c}$. In fact, if $\phi \in$ Aut $_{c} G$ and $A=\langle a\rangle$, then $A^{\beta} \leqslant Z(N) \leqslant N^{\prime} \leqslant$ $\operatorname{ker} \gamma$, and $N^{\gamma} \leqslant\left\langle a^{2^{m-1}}\right\rangle \leqslant\left\langle a^{2}\right\rangle \leqslant \operatorname{ker} \beta$. Thus $\left(\begin{array}{c}\alpha \\ \gamma \delta\end{array}\right)$ and $\left(\begin{array}{c}\alpha^{\prime} \beta^{\prime} \beta^{\prime} \\ \beta^{\prime}\end{array}\right)$ in $\mathscr{H}_{c}$ will commute, provided that (1) $\alpha \beta^{\prime}+\beta \delta^{\prime}=\alpha^{\prime} \beta+\beta^{\prime} \delta$ and (2) $\gamma \alpha^{\prime}+\delta \gamma^{\prime}=\gamma^{\prime} \alpha+$ $\delta^{\prime} \gamma$ hold.

Now $a^{\alpha}=a^{i}, i$ odd, so $a^{\alpha \beta^{\prime}}=a^{\beta^{\prime}}$, since $Z(N)$ has exponent 2 ; moreover $a^{\beta \delta^{\prime}}=a^{\beta}$, since $\delta^{\prime}$, being central, fixes $N^{\prime} \geqslant Z(N)$. Thus $\alpha \beta^{\prime}+\beta \delta^{\prime}=\beta^{\prime}+\beta=$ $\alpha^{\prime} \beta+\beta^{\prime} \delta$, so (1) holds. If $n \in N$, then $n^{\gamma \alpha^{\prime}}=n^{\gamma}$, since $\left|N^{\gamma}\right| \leqslant 2$, and so $N^{\gamma}$ is 
fixed by $\alpha^{\prime}$; moreover, $n^{\delta \gamma^{\prime}}=n^{\gamma^{\prime}}$, since $\delta$ is central, and since $Z(N) \leqslant \operatorname{ker} \gamma^{\prime}$. Thus $\gamma \alpha^{\prime}+\delta \gamma^{\prime}=\gamma+\gamma^{\prime}=\gamma^{\prime} \alpha+\delta^{\prime} \gamma$, so (2) holds. Hence Aut ${ }_{c} G$ is abelian.

EXAmple 3.2. The dihedral group $N$ of order $2^{n+1}, n \geqslant 2$, in which Aut ${ }_{c} N$ is the Klein 4-group, satisfies conditions (i) and (iii) of Theorem 3.1.

COROllary 3.3. If $A$ is cyclic of order $2^{m}, m \geqslant 2$, and if $N$ is a special 2-group, then Aut ${ }_{c}(A \times N)$ is abelian.

Proof. Aut ${ }_{c} N$ fixes $N^{\prime}=Z(N)$ pointwise and so is abelian.

THEOREM 3.4. Let $G=A \times N$, where $A$ is abelian (and where $N$ is arbitrary). If there is a characteristic subgroup $C$ of $G$ such that $N \geqslant C \geqslant Z(N)$, then Aut $G /$ Aut $_{c} G \approx$ Aut $N /$ Aut $_{c} N$.

Proof. Let $T_{G}:$ Aut $G \rightarrow \operatorname{Aut}(G / Z(G))$ take $\phi$ to $\bar{\phi}$, where $\bar{\phi}$ is the automorphism induced on $G / Z(G)$ by $\phi$. Then $T_{G}$ is a homomorphism with kernel Aut ${ }_{c} G$ and image $\operatorname{Im} T_{G} \approx$ Aut $G /$ Aut $_{c} G$. If $T_{N}$ is defined similarly, we shall show that $\operatorname{Im} T_{G} \approx \operatorname{Im} T_{N}$.

From Lemma 2.3, $f:$ Aut $G \rightarrow \mathscr{H}$ taking $\phi$ to $\left({ }_{y \delta}^{\alpha} \beta\right)$ is a monomorphism, and $\delta \in$ Aut $N$. Thus, define $h: \operatorname{Im} T_{G} \rightarrow \operatorname{Im} T_{N}$ taking $\phi$ to $\bar{\delta}$. If $i$ denotes the obvious isomorphism from $G / Z(G)$ to $N / Z(N)$, then $i \bar{\delta}=\bar{\phi} i$, so $h$ is well defined and injective. Further, $h$ is a surjective homomorphism, and so the result follows.

EXAMPLE 3.5. Theorem 3.4 generalizes the example of [1], which shows that there are nonabelian $p$-groups with nonabelian automorphism groups in which every automorphism is central.

Let $G=A \times M$, where $1 \neq A$ is an elementary abelian 2-group, and where $M$ is the generalized Miller group of Section 1. For $p$ odd, let $G=A \times N$, where $1 \neq A$ is any abelian $p$-group, and where $N$ is the special $p$-group of Jonah and Konvisser [5]. Then by Theorem 3.4, taking $C=\Phi(G)=\Phi(M)=Z(M)$ in the first case and $C=G^{\prime}=N^{\prime}=Z(N)$ in the second, we obtain Aut ${ }_{c} G=$ Aut $G$. But by Theorem 3.1, Aut $G$ is nonabelian.

\section{Semidirect products with abelian automorphism groups}

Throughout this section let $A=\langle a\rangle$ be a cyclic group of order $2^{m}, m \geqslant 3$. If $G=A N$ is a nontrivial semidirect product, then any automorphism of $A$ can be extended to an automorphism of $G$ which acts trivially on $N$. Thus, if Aut $G$ is abelian and $\phi: a \rightarrow a^{3}$, then $\phi \in$ Aut $_{c} G$, so $a^{2} \in Z(G)$ and is fixed by the action 
of $N$. Hence $N$ has a subgroup $J$ of index 2 such that

$$
a^{n}=a \quad \text { if } n \in J \quad \text { and } \quad a^{n}=a^{1+2^{m-1}} \quad \text { if } n \in N \backslash J .
$$

Conversely, suppose for the remainder of the section that $N$ is a nonabelian special 2-group with a subgroup $J$ of index 2 and that $G$ is the semidirect product defined by (*). Then Theorem 4.2 gives conditions for Aut $G$ to be abelian, but first we establish some properties of $G$.

LEMMA 4.1. If $G=A N$ is defined as above, then

(i) $Z(N)<J$

(ii) for $\phi \in$ Aut $G,\left[N^{\gamma}, N\right]=1$;

(iii) $\operatorname{Aut}(N: J) \geqslant$ Aut $_{c} G$;

(iv) $A \times J$ is characteristic in $G$.

Proof. (iii) follows from (i), which is obvious.

(ii) Any element in $G$ of the form $a^{2 i+1} n, n \in N$, has order $2^{m}$. Thus $N^{\gamma} \leqslant\left\langle a^{2}\right\rangle$, since $N$ has exponent 4 .

(iv) $C_{G}(a)=A \times J$, so $a$ has 2 conjugates. If $\phi \in$ Aut $G$ and $a^{\phi}=a^{2 i+1} n$, $n \in N$, then $C_{N}(n) \geqslant J$, since $a^{\phi}$ must also have 2 conjugates. Thus $n \in Z(J)$, and so $C_{G}\left(a^{\phi}\right)=A \times J$.

THEOREM 4.2. If $G=A N$ is defined as above, and if

(i) $A \times Z(N)$ is characteristics in $G$, and

(ii) $\operatorname{Aut}(N: J)=\operatorname{Aut}_{c} N$,

then Aut $G$ is abelian.

Proof. Aut $G$ is isomorphic to a subgroup of $\operatorname{Aut}(A \times N)$ by Corollary 2.6, since the conditions of Theorem 2.4 follow from (i) and from Lemma 4.1. But if $\phi \in$ Aut $G$, then $\delta \in \operatorname{Aut}_{c} N$, for otherwise, by (ii), there is some $j \in J$ with $j^{\delta} \notin J$. Thus $(1, j)^{\phi}=\left(j^{\gamma}, j^{\phi}\right) \notin A \times J$, contradicting Lemma 4.1(iv). Hence, by Theorem 2.5(ii), Aut $G$ is isomorphic to a subgroup of Aut ${ }_{c}(A \times N)$, which is abelian by Corollary 3.3. (In fact from Sanders' order formula [7], $\left|A \mathrm{At}_{\mathrm{c}} G\right|=$ $\mid$ Aut ${ }_{c}(A \times N) \mid$, so Aut $G \approx$ Aut $_{c}(A \times N)$.)

ExAmple 4.3. Let $M=A N$ be the generalized Miller group of Section 1. Then $A \times J=\left\langle a, b^{2}, c\right\rangle$ is characteristic in $G$, and $\langle a, b\rangle$ is also, since it is the only subgroup of index 2 in $M$ which is of type $\left\langle x, y \mid x^{2^{m}}=y^{4}=1, x^{y}=x^{1+2^{m-1}}\right\rangle$. Thus $A \times Z(N)=\left\langle a, b^{2}\right\rangle=\left\langle a, b^{2}, c\right\rangle \cap\langle a, b\rangle$ is characteristic in $M$. Further, Aut ${ }_{c} N=\operatorname{Inn} N$ is of index 2 in Aut $N \approx D_{4}$, and since $J$ is not characteristic, $\operatorname{Aut}(N: J)=\operatorname{Aut}_{c} N$. Thus Aut $M$ is abelian (which is the result of [8]). 
COROLlary 4.4. If $G=A N$ is defined as above, and if

(i) $Z(J)=Z(N)$, and

(ii) $\operatorname{Aut}(N: J)=\operatorname{Aut}_{c} N$,

then Aut $G$ is abelian.

Proof. As in Lemma 4.1(iv), if $\phi \in$ Aut $G$ and $a^{\phi}=a^{2 i+1} n, n \in N$, then $C_{N}(n) \geqslant J$, so $C_{N}(n)=J$ or $C_{N}(n)=N$. In either case, $n \in Z\left(C_{N}(n)\right)=Z(N)$. So $A^{\beta} \leqslant Z(N)$, and condition (i) of Theorem 4.2 follows.

ExAmples 4.5. We use the notation of the Hall and Senior tables [4], in which the index $\mid$ Aut $N$ : Aut ${ }_{c} N \mid$ is denoted $t_{3}$. Aut $N /$ Aut $_{c} N$ acts on the subgroups $\Omega$ of index 2 in $N$, and if the orbit of $J \in \Omega$ has length $t_{3}$, then $\operatorname{Aut}(N: J)=\operatorname{Aut}_{c} N$. In particular, this holds if $t_{3}$ is prime and $J$ is not characteristic.

The families $32 \Gamma_{4}$ and $64 \Gamma_{9}$ of the tables are nonabelian special 2-groups (of orders 32 and 64, respectively). Further, if $N$ lies in either of these families, then any non-characteristic subgroup $J$ of index 2 is of type $\Gamma_{2}$, and so $Z(J)=Z(N)$. The table below lists the group $N$, the parameter $t_{3}$, and the choice for $J$ (up to isomorphism) which yields a group $G=A N$ with Aut $G$ abelian. Further examples can be found in the families $64 \Gamma_{10}$ and $64 \Gamma_{11}$.

\begin{tabular}{|c|c|c|c|c|c|c|}
\hline$N$ type $\Gamma_{4}$ & $b_{1}$ & $b_{2}$ & $c_{1}$ & $c_{2}$ & $c_{3}$ & $d$ \\
$t_{3}$ & 2 & 2 & 2 & 4 & 4 & 3 \\
$J$ type $\Gamma_{2}$ & $c_{1}, a_{1}$ & $c_{1}, c_{2}$ & $c_{1}, c_{2}$ & $c_{1}$ & $c_{2}$ & $c_{1}, c_{2}$ \\
\hline$N$ type $\Gamma_{9}$ & $b_{1}$ & $b_{4}$ & $c$ & $d_{1}$ & $d_{2}$ & \\
$t_{3}$ & 2 & 2 & 3 & 2 & 2 & \\
$J$ type $\Gamma_{2}$ & $c_{1}$ & $h, c_{2}$ & $c_{1}, c_{2}$ & $h, c_{1}$ & $h$ & \\
\hline
\end{tabular}

\section{References}

[1] M. J. Curran, 'A non-abelian automorphism group with all automorphisms central', Bull. A ustral. Math. Soc. 26 (1982), 393-397.

[2] J. D. Dixon, Problems in group theory (Dover Publications, New York, 1973).

[3] B. E. Earnley, On finite groups whose group of automorphisms is abelian (Ph.D. thesis, Wayne State University, 1975).

[4] M. Hall, Jr. and J. K. Senior, The groups of order $2^{n},(n \leqslant 6)$ (New York, 1964).

[5] D. Jonah and M. Konvisser, 'Some non-abelian p-group with abelian automorphism groups', Arch. Math. 26 (1975), 131-133. 
[6] G. A. Miller, 'A non-abelian group whose group of isomorphisms is abelian', Messenger of Math. 43 (1913), 124-125.

[7] P. R. Sanders, 'The central automorphisms of a finite group', J. London Math. Soc. 44 (1969), 225-228.

[8] R. R. Struik, 'Some non-abelian 2-groups with abelian automorphism groups', Arch. Math. 39 (1982), 299-302.

\section{Department of Mathematics}

University of Otago

Dunedin

New Zealand 